\title{
THE IMPORTANCE OF JUMPS IN PRICING
}

\section{EUROPEAN OPTIONS}

\author{
F. Campolongo $^{(1) 1 *}$, J. Cariboni ${ }^{(1),(2)}$, and W. Schoutens ${ }^{(2)}$
}

1. European Commission, Joint Research Centre, Via Fermi 1, Ispra 21020, Italy

2. K.U.Leuven, U.C.S., W. De Croylaan 54, B-3001 Leuven, Belgium

\begin{abstract}
The screening method proposed by Morris [1] and recently improved by Campolongo et al. [2] has been employed to estimate the importance of the inclusion of jumps in a model for pricing European options. Results confirm that, among the sources of uncontrollable uncertainty, jumps play a major role and therefore need to be better investigated in order to improve the accuracy of the model predictions.
\end{abstract}

Keywords: option pricing, Heston model, jumps, sensitivity analysis, Morris method, variance based sensitivity indices.

\section{SETTING THE PROBLEM}

Imagine an investor enters into a European call option contract. A European call option is a contract that gives the holder the right (not the obligation) to buy an underlying asset by a certain date for a certain price. The price in the contract is known as the exercise price or strike price; the date in the contract is known as the expiration date or maturity. To enter into an option contract there is a cost

\footnotetext{
* Corresponding author: F. Campolongo. E-mail: francesca.campolongo@jirc.it; Ph: +39(0332)785476; Fax: $+39(0332) 785733$
} 
corresponding to the right purchased. This cost, referred to as premium, is the price of the option at present day.

The option price is established according to the theory of arbitrage-free pricing. Classic deterministic arbitrage involves buying an asset at a low price in one market whilst immediately selling it at a higher price in another market to make a risk-free profit. The theory of arbitrage-free prices imposes that the prices of different instruments must be related to one another in such a way that they offer no arbitrage opportunities. In practice to price the option we make use of a model describing the evolution through time of the underlying asset price and then impose no arbitrage arguments.

The risk associated with an option contract derives from the unknown evolution of the underlying price on the market. This risk is not reducible and is an intrinsic feature of the contract itself. Apart from this risk, neither controllable nor reducible, there is another part of risk which is that deriving from the fact that the current option price is an estimated quantity, potentially affected by an error. If for instance the call option price is overestimated, the option holder faces the risk of losing more money than what he should (in case of loss). The more accurate the price estimate, the less the risk associated with the option.

The current option price is calculated via a mathematical model (describing the evolution of the underlying) that contains a number of input variables whose values are affected by uncertainty. The problem addressed in this paper is that of quantifying the uncertainty affecting the current option price, identifying its main sources and provide indication on how to reduce the "controllable" risk. In particular, we aim to assess the importance of incorporating the effects of jumps in modelling the underlying dynamics. The model chosen for describing the underlying stock price evolution is the Heston Stochastic Volatility model (HEST, [3]) with jumps [4], where the stock price follows the Black-Scholes stochastic differential equation SDE in which the volatility behaves stochastically over time and jumps are included in the dynamics of the asset. Different scenarios are assumed, corresponding to different possible strike prices and times to maturity.

Results show that jumps drive most of the uncertainty in the estimated option price, thus confirming their key role in the pricing process. The importance of jumps is more evident for higher strike prices. 


\section{THE HESTON STOCHASTIC VOLATILITY MODEL WITH JUMPS}

In [3] Heston introduced a stock price model which introduced stochastic volatility in the famous Black Scholes [5]. In the Heston model the price paths of a stock (and the volatility) are continuous. Later, extensions of the Heston model were formulated that also allowed for jumps in the stock price paths. We investigate here this extension and ask ourself the question whether the introduction of jumps leads to a significant increase in the variance of the prices of derivatives under this model.

We denote the stock price process by $S=\left\{S_{t}, t \geq 0\right\}$ and assume that the stock pays out a continuous compound dividend yield of $q \geq 0$. Moreover, we assume in our market we have a riskless bank account available, paying out a continuous interest rate $r$.

In the Heston Stochastic Volatility model with jumps (HESJ) the stock price process is a modelled by a SDE given by:

$$
\frac{d \mathrm{~S}_{t}}{d t}=\left(r-q-\lambda \mu_{J}\right) d t+\sigma_{t} d W_{t}+\mathrm{J}_{t} d \mathrm{~N}_{t} \quad S_{0} \geq 0
$$

where $\mathrm{N}=\left\{\mathrm{N}_{t}, t \geq 0\right\}$ is an independent Poisson process with intensity parameter $\lambda>0$, i.e. $E\left[\mathrm{~N}_{t}\right]=\lambda t . \mathrm{J}_{\mathrm{t}}$ is the percentage jump size (conditional on a jump occurring) that is assumed to be log-normally, identically and independently distributed over time, with unconditional mean $\mu_{J}$.

The standard deviation of $\log \left(1+\mathbf{J}_{t}\right)$ is $\sigma_{J}$ :

$$
\log \left(1+\mathrm{J}_{t}\right) \sim \mathrm{N}\left(\log \left(1+\mu_{j}\right)-\frac{\sigma_{J}^{2}}{2}, \sigma_{J}^{2}\right)
$$

The (squared) volatility follows the classical Cox-Ingersoll-Ross (CIR) process:

$$
d \sigma_{t}^{2}=k\left(\eta-\sigma_{t}^{2}\right) d t+\theta \sigma_{t} d \tilde{W}_{t} \quad \sigma_{0} \geq 0
$$

where $W=\left\{W_{t}, t \geq 0\right\}$ and $\tilde{W}=\left\{\tilde{W}_{t}, t \geq 0\right\}$ are two correlated standard Brownian motions such that $\operatorname{Cov}\left[d W_{t}, d \tilde{W}_{t}\right]=\rho d t$. Finally, $\mathbf{J}_{\mathrm{t}}$ and $N$ are independent, as well as of $W_{t}$ and $\tilde{W}_{t}$.

The characteristic function $\phi(u, t)$ is in this case given by: 


$$
\begin{aligned}
\phi(u, t)= & E\left\{\exp \left(\mathrm{i} u \log \left(S_{t}\right) \mid S_{0}, \sigma_{0}^{2}\right\}=\right. \\
= & \exp \left(\mathrm{i} u\left(\log S_{0}+(r-q) t\right)\right) \\
& \times \exp \left(\eta \kappa \theta^{-2}\left((\kappa-\rho \theta u \mathrm{i}-d) t-2 \log \left(\left(1-g e^{-d t}\right) /(1-g)\right)\right)\right) \\
& \times \exp \left(\sigma_{0}^{2} \theta^{-2}\left((\kappa-\rho \theta u \mathrm{i}-d)\left(1-e^{-d t}\right) /\left(1-g e^{-d t}\right)\right)\right. \\
& \times \exp \left(-\lambda \mu_{J} t u \mathrm{i}+\lambda t\left(\left(1+\mu_{J}\right)^{u \mathrm{i}} \exp \left(\sigma_{J}^{2}(u \mathrm{i} / 2)(u \mathrm{i}-1)\right)-1\right)\right)
\end{aligned}
$$

where

$$
\begin{aligned}
& d=\left((\rho \theta u \mathrm{i}-\kappa)^{2}-\theta^{2}\left(-u \mathrm{i}-u^{2}\right)\right)^{1 / 2}, \\
& g=(\kappa-\rho \theta u \mathrm{i}-d) /(\kappa-\rho \theta u \mathrm{i}+d) .
\end{aligned}
$$

Pricing of European call options under this model can be done by the Carr and Madan [6] pricing method. This method is applicable for classical vanilla options when the characteristic function of the risk-neutral stock price process is known.

Let $\alpha$ be a positive constant such that the $\alpha$-th moment of the stock price exists. For all stock price models

encountered here, typically a value of $\alpha=0.75$ will do fine. Carr and Madan then showed that the price $C(K, T)$ of a European call option with strike $K$ and time to maturity $T$ is given by:

$$
C(K, T)=\frac{\exp (-\alpha \log (K))}{\pi} \int_{0}^{+\infty} \exp (-\mathrm{i} v \log (K)) \rho(v) d v,
$$

where

$$
\begin{aligned}
\rho(v) & =\frac{\exp (-r T) E\left[\exp \left(\mathrm{i}(v-(\alpha+1) \mathrm{i}) \log \left(S_{T}\right)\right)\right]}{\alpha^{2}+\alpha-v^{2}+\mathrm{i}(2 \alpha+1) v} \\
& =\frac{\exp (-r T) \phi(v-(\alpha+1) \mathrm{i}), T)}{\alpha^{2}+\alpha-v^{2}+\mathrm{i}(2 \alpha+1) v}
\end{aligned}
$$

Using Fast Fourier Transforms, one can compute within a second the complete option surface on an ordinary computer.

\section{THE SENSITIVITY ANALYSIS METHODOLOGY}

A sensitivity analysis method very efficient in identifying important factors in a model with just very few model evaluations is the design proposed by Morris [1]. The method requires a total number of 
model evaluations which is a linear function of the number of input factors involved, and it does not rely on strict assumptions about the model, such as for instance additivity or monotonicity of the model input-output relationship.

The Morris method has several desirable features of a sensitivity analysis methodology. It is conceptually simple, easy to implement, and its results are easily interpreted. It can cope with the influence of scale and shape because it is sensitive not only to the effect of the ranges of input variation but also to the form of their probability density function (pdf). It can be regarded as a global method (in contrast to the derivative-based ones) as the final sensitivity measure is obtained by averaging a number of local measures (the elementary effects), computed at different points of the input space.

In very recent work [2] Campolongo and coworkers proposed an improved version of the Morris measure $\mu$, denoted as $\mu^{*}$, which is more effective in ranking factors in order of importance.

Furthermore, the new measure could be extended to the capability to treat groups of factors as if they were single factors, another desirable property of a sensitivity analysis.

In this work we employ the new measure $\mu^{*}$ to assess the sensitivity of an option price to the several uncertain factors determining its value; and in particular focus our attention on the importance of introducing jumps in the dynamics of the underlying asset. As a further confirmation of the reliability of the new measure, results obtained according to $\mu^{*}$ are also compared with those obtained through the use of the variance based methods. Variance based methods have all the desirable properties mentioned above and the further great advantage of an easy interpretation in terms of output variance decomposition. A variance based method estimates the percentage of output variance that each factor is accounting for. However, as a drawback, these methods require a computational effort that in some instances may be prohibitive, as the number of model evaluations required is almost 100 times higher than that for the Morris strategy.

\section{The Morris method and its improved version}

The experimental plan proposed by Morris [1] is composed of individually randomized 'one-factor-at-atime' experiments: the impact of changing one factor at a time is evaluated in turn. Each input factor may assume a discrete number of values, called levels, which are chosen within the factor range of variation. 
The sensitivity measures proposed in the work of Morris are based on what is called an elementary effect. The elementary effect for the $i$ th input is defined as follows. Let $\Delta$ be a predetermined multiple of $1 /(p-1)$. For a given value of $\mathbf{x}$, the elementary effect of the $i$ th input factor is defined as

$$
E E_{i}(\mathbf{x})=\frac{\left[y\left(x_{1}, . ., x_{i-1}, x_{i}+\Delta, x_{i+1}, . ., x_{k}\right)-y(\mathbf{x})\right]}{\Delta}
$$

where $\mathbf{x}=\left(x_{1}, x_{2}, \ldots, x_{k}\right)$ is any selected value in $\Omega$ such that the transformed point $\left(\mathbf{x}+\mathbf{e}_{i} \Delta\right)-\mathbf{e}_{i}$ being a vector of zeros but with a unit as its $i$ th component - is still in $\Omega$ for each index $i=1, . ., k$. The finite distribution of elementary effects associated with the $i$ th input factor, is obtained by randomly sampling different $\mathbf{x}$ from $\Omega$, and is denoted by $F_{i}$.

In Morris [1] two sensitivity measures were proposed for each factor: $\mu$, an estimate of the mean of the distribution $F_{i}$, and $\sigma$, an estimate of the standard deviation of $F_{i}$. A high value of $\mu$ indicates an input factor with an important overall influence on the output. A high value of $\sigma$ indicates a factor involved in interactions with other factors or whose effect is non-linear. In Campolongo et al. [2] we consider a third sensitivity measure, $\mu^{*}$, which is an estimate of the mean of the distribution, here denoted as $G_{i}$, of the absolute values of the elementary effects. For non monotonic models, the measure $\mu^{*}$ performs better than $\mu$ [2]. In fact, if the distribution $F_{i}$ contains elements of opposite sign, which occurs when the model is non-monotonic, when computing its mean some effects may cancel each other out. Thus a factor which is important but whose effect on the output has an oscillating sign may be erroneously considered as negligible, generating a mostly undesirable Type II error.

\section{The variance based measures}

Variance based methods choose as a measure of the main effect of a factor $X_{i}$ on the output, an estimation of quantity $\frac{V_{X_{i}}\left(E_{\mathbf{X}_{-i}}\left(Y \mid X_{i}\right)\right)}{V(Y)}$, which is known in the literature as the "first order effect" of $X_{i}$ on $Y$, and denoted by $S_{i}$. Reasons for this choice are detailed in [7]. 
Another sensitivity measured based on the variance decomposition is the total sensitivity index, $S_{T_{i}}$, which estimates the sum of all effects involving a given factor $X_{i} . S_{T i}$ is estimated by the quantity $\frac{E_{\mathbf{X}_{-i}}\left(V_{X_{i}}\left(Y \mid \mathbf{X}_{-i}\right)\right)}{V(Y)}$. The total index is the appropriate measure to use when the goal is that identifying irrelevant factors, i.e. those that can be fixed to any given value within their range of variation because they are non influent on the total output variance. A necessary and sufficient condition for factor $X_{i}$ to be totally non-influent is that $S_{T i}=0$. In fact, if factor $X_{i}$ is totally non-influent, then all the variance is due to $\mathbf{X}_{-i}$, and fixing this vector results in $V_{X_{i}}\left(Y \mid \mathbf{X}_{-i}\right)=0$, as well as in $E_{\mathbf{X}_{-i}}\left(V_{X_{i}}\left(Y \mid \mathbf{X}_{-i}\right)\right)=0$. The reverse is also true: if $V_{X_{i}}\left(Y \mid \mathbf{X}_{-i}\right)=0$ at all fixed points in the space of $\mathbf{X}_{-i}$, then $X_{i}$ is non-influent, so that $S_{T i} \equiv 0$.

Although very accurate and reliable, the variance based measures have a disadvantage in their computational cost. These methods require a number of model evaluation which is $N \times(k+2)$, where $k$ is the number of input factors and $N$ is of the order of $N=500,5000 \ldots$, [7]. When $k$ is large or the model is very time consuming the required computational time may be unaffordable.

\section{THE SENSITIVITY ANALYSIS EXPERIMENT}

The sensitivity measure $\mu^{*}$ proposed in [2] is employed here to assess the importance of the introduction of jumps in the dynamics of the stock price. Furthermore, as a further confirmation of the reliability of this measure, we compute the variance based indices $\left\{\mathrm{S}_{i}, \mathrm{~S}_{T_{i}}\right\}$ and compare them with the Morris results. Note that the computational cost to estimate the variance based indices in this case is not a problem as the model under examination is not excessively time consuming.

The input factors selected for sensitivity analysis purposes are listed in Table 1 with the correspondent distributions. The input factors in Table 1 can be distinguished in two groups: those whose value can be estimated with a certain degree of confidence by looking at real data, and therefore represent a source of 
uncertainty that can be defined as "controllable"; and those that cannot be checked with market data and are therefore regarded as completely "uncontrollable". The first group consists of the initial condition for the dynamics of the volatility $\sigma_{0}$, the dividend yield $q$ and the interest rate $r$. The remaining inputs, among which the jumps parameters, belong to the second group.

The Morris measure $\mu^{*}$, the first sensitivity indices $S_{i}$ and total sensitivity indices $S_{T_{i}}$ are computed for each input factor in 42 different scenarios, a scenario being determined by a different value of the option strike price and of the time to maturity. Seven values of the strike price are considered (strike price $=70,80, \ldots, 130$ ) to represent situations in which the option is in the money, at the money or out of the money (the initial condition for the stock is fixed at $\mathrm{S}_{0}=100$ ). Six different time horizons are examined from 0.5 years up to 3 years.

The total number of model executions to estimate the entire set of the variance based indices $\left\{\mathrm{S}_{i}, \mathrm{~S}_{T_{i}}, i=1,2, \ldots 10\right\}$ is $N_{V B}=24.576$. To estimate the revised Morris measure $\mu^{*}, 4$ possible levels are considered for each input and a total number of 110 model executions are performed. In order to compare results the Morris $\mu^{*}$ and the total sensitivity indices $S_{T_{i}}$, that normally do not sum up to 1 , are rescaled in $[0,1]$ (see Figures 1 and 2). In the figures each graph refers to a fixed input. Within a graph, each dot illustrates the input importance in a specific scenario: the bigger the dot size the higher the importance of the input in the scenario. Ten different dots' sizes are considered corresponding to ten different classes of importance, ranging from sensitivity measure values in $[0,0.1]$ to sensitivity measure values in $[0.9,1]$.

Fixing a factor, the comparison between the graph of Figure 1 and that of Figure 2 allows evaluating the effectiveness of $\mu^{*}$ in measuring the factor importance in different scenarios. The Morris design is confirmed to be a good proxy of the total sensitivity index (see also [2]). Minor improvements in the correspondence of results are achieved by increasing the Morris sample size up to $N_{\text {Morris }}=1.100$.

Results highlight that, overall (i.e. not focusing on a selected scenario), the most influential parameters are: the dividend yield $q$, the interest rate $r$, and the jump parameters $\lambda$ and $\sigma_{\mathrm{j}}$. In particular, $q$ and $r$ are very important for low strike prices at all times to maturity, while $\lambda$ and $\sigma_{\mathrm{j}}$ are more relevant at higher 
strike prices. As expectable, $\sigma_{0}$ is important only for low times to maturity, especially when the option is at the money.

Note that among these 4 most important factors, $q$ and $r$ belong to the group of these that can be considered as "controllable". More interesting is the role of the jumps parameters $\lambda$ and $\sigma_{\mathrm{j}}$ that are the most relevant among the "uncontrollable" factors. In general, if we restrict our attention to the "uncontrollable" factors, it turns out that is the jumps group, i.e. $\lambda, \sigma_{\mathrm{j}}$ and $\mu_{\mathrm{j}}$, which drives the higher amount of the total output variance in all scenarios.

If we compare the relative importance of the various factors in different scenarios, it emerges that it is rather stable with respect to shifts in the time horizon, while it varies substantially by changing the strike price value.

In Figure 4 we represent three scenarios that correspond to 3 different strike prices: we consider an option in the money $($ strike $=70)$, at the money $($ strike $=100)$, and out of the money $($ strike $=130)$, with a time to maturity fixed at 1.5 years. In each pie the total variance of the option price is apportioned to the contributions due to the first order effects of each input (i.e. the Sobol' $\mathrm{S}_{i}$ ) and to the interactions of all orders. Since interactions do not explain a high amount of the variance they are summed up in a single term. The figures stresses that the importance of jumps increases considerably with the strike price. The sum of their main effects goes from $4 \%$, when the option is in the money, to $29 \%$ when the option is at the money, up to $45.3 \%$ when the strike price reaches 130 (option out the money). When the option is in the money more than $90 \%$ of the total variance is due to the controllable factors $q$ and $r$, thus leaving few chances to reduce the uncertainty in the option price by increasing our modelling effort. As the strike price increases the importance of jumps augments considerably, making evident that their role in modelling the option price can not be overlooked: jumps need to be included in the model and their representation should be as much accurate as possible. 


\section{CONCLUSIONS}

In this work we have employed the revised version of the sensitivity measure proposed by Morris to estimate the importance of the inclusion of jumps in a model for pricing European options: the Heston model.

The recently revised sensitivity measure confirmed its capability to distinguish between important and negligible input factors at low computational cost.

Concerning the model, the sensitivity analysis has led to the conclusion that among the "uncontrollable" factors, i.e. those that can not be estimated from market data, jumps play a major role in determining the option price, thus stressing the need of including them in the model formulation.

If we consider all factors, than at low strike prices most of the uncertainty in the option price is due to "controllable" factors such as $q$ and $r$. As the option strike price increases, the importance of jumps increases considerably: for instance for option with strikes 120 and 130, the importance of jumps is superior to that of $q$ and $r$ for all times to maturity. This underlines that an accurate assessment of the jump process becomes more urgent for out of the money options.

A final remark is that, as expectable, at low time to maturity the initial condition for volatility needs to be accurately determined while its importance decreases as the time to maturity increases.

\section{REFERENCES}

[1] M. D. Morris, Factorial Sampling Plans for Preliminary Computational Experiments, Technometrics, 1991, 33, 161-174.

[2] F. Campolongo, J. Cariboni, and A. Saltelli. Sensitivity analysis: the Morris method versus the variance based measures, 2003, submitted to Technometrics.

[3] Heston, S., A closed-form solution for options with stochastic volatility with applications to bond and currency options, Review of Financial Studies, 1993, 6, 327-343.

[4] Bakshi, G., Cao, C. and Chen, Z., Empirical Performance of Alternative Option Pricing Models, The Journal of Finance, 1997, Vol. LII, No. 5, 2003-2049. 
[5] Black, F. and Scholes, M., The pricing of options and corporate liabilities, Journal of Political Economy, 1973, 81, 637-654.

[6] Carr, P. and Madan, D., Option Valuation using the Fast Fourier Transform, Journal of Computational Finance, 1998, 2, 61-73.

[7] A. Saltelli, S. Tarantola, F. Campolongo, M. Ratto. Sensitivity Analysis in Practice. A Guide to Assessing Scientific Models, 2004. John Wiley \& Sons publishers, Probability and Statistics series.

\section{VITAE}

Francesca Campolongo

Jessica Cariboni attained her degree in physics at the University of Milan (Italy) in 2000. After two years working as quant analyst in Nextra Investment Management SgR (Banca Intesa), she obtained a grant from the European Commission in January 2003 to start a $\mathrm{PhD}$ at the Department of Mathematics of the Katholieke Universiteit Leuven (Belgium).

Wim Schoutens

\section{FIGURE CAPTIONS}

Table 1: Distributions for the inputs of the Heston model.

Figure 1: Importance of the factors in each of the 42 scenarios according to $\mu^{*}$ rescaled in $[0,1]$. The differences in the size of the dots represent the differences in the importance of the fixed input factors in the scenarios.

Figure 2: Importance of the factors in each of the 42 scenarios according to $S_{T i}$ rescaled in [0,1]. The differences in the size of the dots represent the differences in the importance of the fixed input factors in the scenarios.

Figure 3: Decomposition of the total variance of the strike price in three scenarios. Interactions of all orders are grouped in a single term.

\section{Table 1}




\begin{tabular}{|c|c|c|c||c|c|c|c|}
\hline Input & Distribution & Minimum & Maximum & Input & Distribution & Minimum & Maximum \\
\hline$\sigma_{0}$ & Uniform & 0.04 & 0.09 & $\lambda$ & Uniform & 0 & 2 \\
\hline$\kappa$ & Uniform & 0 & 1 & $\mu \mathrm{j}$ & Uniform & -0.1 & 0.1 \\
\hline$\eta$ & Uniform & 0.04 & 0.09 & $\sigma \mathrm{j}$ & Uniform & 0 & 0.2 \\
\hline$\theta$ & Uniform & 0.2 & 0.5 & $\mathrm{r}$ & Uniform & 0 & 0.05 \\
\hline$\rho$ & Uniform & -1 & 0 & $\mathrm{q}$ & Uniform & 0 & 0.05 \\
\hline
\end{tabular}

Figure 1: Morris r=10 

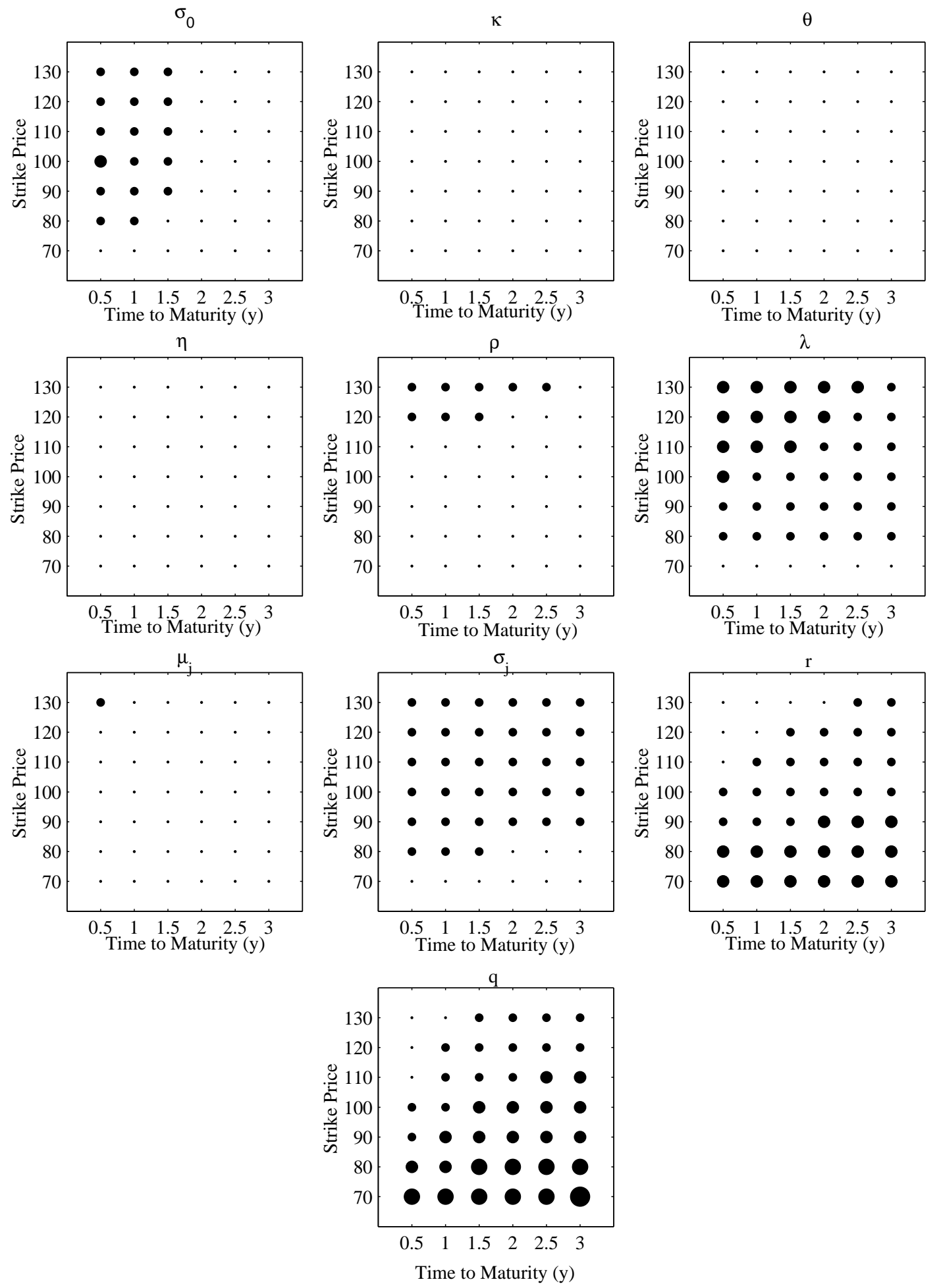

Figure 2: Sobol 

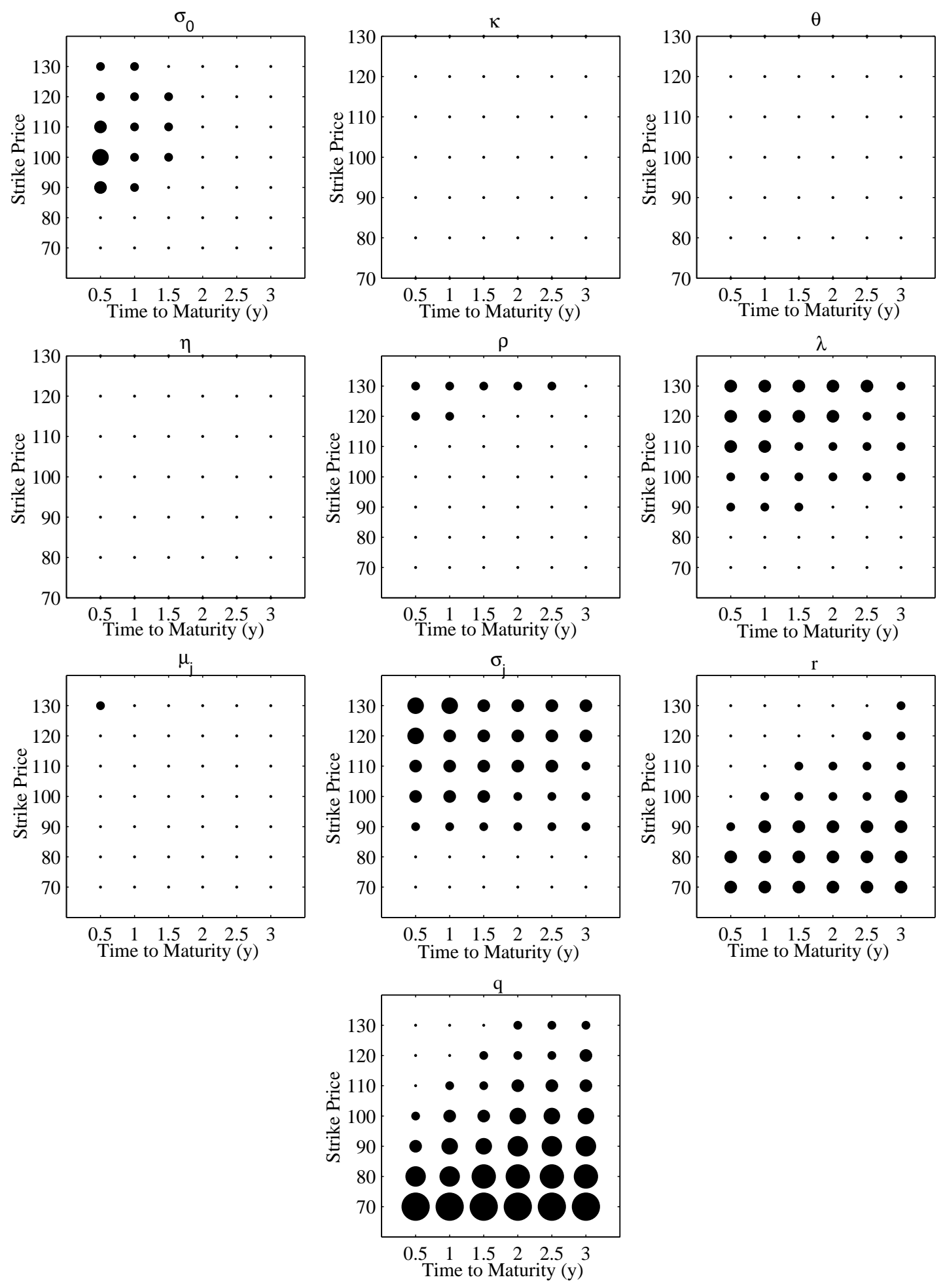

Figure 3 
Strike Price $=70$, Time to Maturity $=1.5 \mathrm{y}$

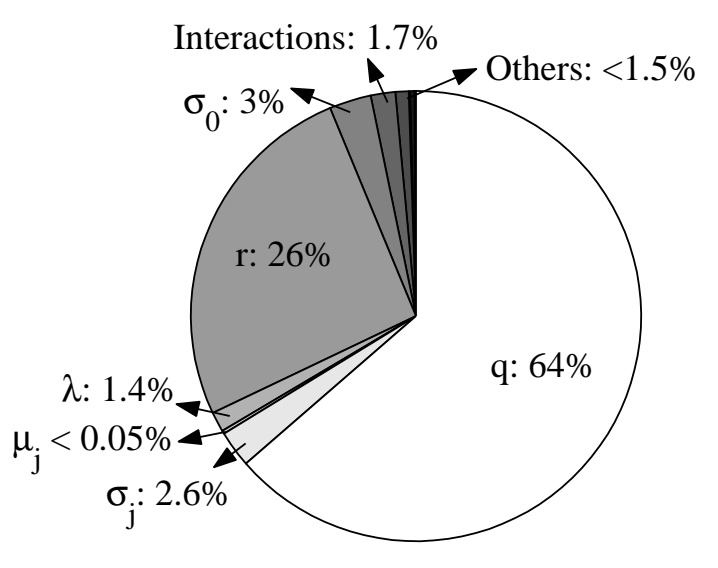

Strike Price=130, Time to Maturity $=1.5 y$

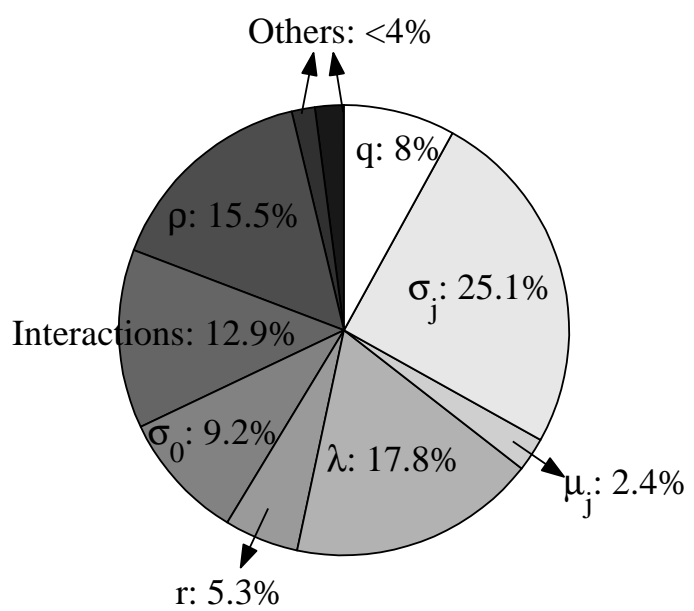

Strike Price=100, Time to Maturity $=1.5 y$

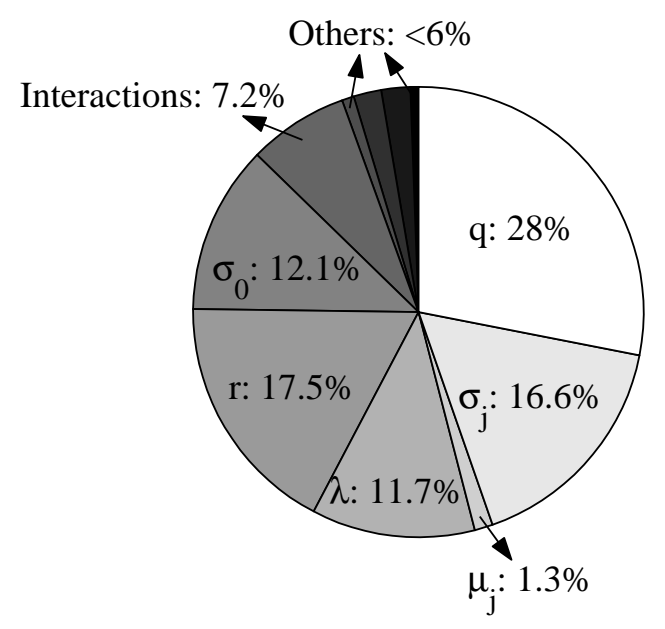

